\title{
79. Some Dominated Convergence Theorems in a von Neumann Algebra
}

\author{
By A. R. Padmanabhan \\ Indian Statistical Institute, Calcutta \\ (Comm. by Kinjirô KunUGI, M.J.A., April 12, 1966)
}

Summary. In his fundamental paper [2], Stinespring proved several dominated convergence theorems for operators measurable w.r.t. a gage space. In this paper we state and prove some dominated convergence theorems. One of our theorems is a generalisation of a theorem of Stinespring [2]. In the others, we obtain some results under assumptions, which are weaker than those of Stinespring.

Throughout this paper, the notation and terminology will be the same as those of Segal [1] and Stinespring [2]. Let $(H, \Omega, m)$ be a regular gage space, namely, $H$ is a complex Hilbert Space, $\Omega$ a ring of operators (=von Neumann Algebra) acting on $H$ and $m$, a gage on $\Omega$, such that for any projection $P, m(P)=0$ implies $P=0$. Denote the $L^{1}$ and $L^{2}$-space of the gage space by $L^{1}(H, \Omega, m)=L^{1}(\Omega, m)$ and $L^{2}(H, \Omega, m)=L^{2}(\Omega, m)$, respectively. A sequence of measurable operators (measurable w.r.t. $\Omega$ in the sense of $[1]$ ), is said to converge grossly to a measurable operator $A,[2, \mathrm{p} .26]$, if, for every $T$ in $L^{1}(\Omega, m)$, and for every $\varepsilon>0$, there exists a positive integer $N$ such that for all $n \geqq N$, there exists a projection $P_{n}$ with the property that $\left\|\left(A_{n}-A\right) P_{n}\right\|<\varepsilon$ and $|m(T Q)|<\varepsilon$ for any projection $Q \lesssim I-P_{n}$ $(Q$ in $\Omega)$. Our definition of convergence in measure will be the same as that of Stinespring $[2$, p. 23]. It is known $[2$, p. 27] that convergence in measure always implies gross convergence.

Let $\left(A_{n}\right)$ be a sequence of measurable operators converging grossly to a measurable operator $A$. Suppose there exists an operator $B$ in $L^{1}(\Omega, m)$, such that $\left|A_{n}-A\right| \leqq B, n=1,2, \cdots$. From a dominated convergence theorem of Stinespring [2, Theorem 4.6] it follows that $A_{n}-A \rightarrow 0$ in $L^{1}(\Omega, m)$, hence in particular $m\left(A_{n}\right) \rightarrow m(A)$.

However, there are cases, which are not covered by this Theorem. We give below an example of a sequence $\left(A_{n}\right)$ of non-negative, integrable operators converging grossly to zero (the zero operator) and $m\left(A_{n}\right) \rightarrow m(0)=0$, but there does not exist any integrable operator $T$ with $A_{n} \leqq T$ for all $n$. Let $\Omega$ be a continuous finite factor and $m$, the standard faithful normal trace on $\Omega$ with $m(I)=1$. Let $P_{1}$ be a projection with $m\left(P_{1}\right)=1 / 2$. Let $P_{2}$ be a projection contained in $I-P_{1}$ with $m\left(P_{2}\right)=1 / 2^{2}, \cdots$, and in general $P_{n}$ a projection contained 
in $I-P_{1}-P_{2}-\cdots-P_{n-1}$ with $m\left(P_{n}\right)=1 / 2^{n}$. Let $A_{n}=2^{n} / n P_{n}$. Each $A_{n}$ is integrable and $\left(A_{n}\right)$ converges in measure and hence grossly to zero and $m\left(A_{n}\right)=1 / n \rightarrow 0=m(0)$. If possible let there exist a non-negative measurable operator $T$ such that $A_{n} \leqq T$ for all $n$. Then easy to see that $m(T) \geqq m\left(T P_{1}\right)+\cdots+m\left(T P_{n}\right)=\sum_{k=1}^{n} \frac{1}{k}$ for all $n$, which shows that $T$ cannot be integrable. In order to cover such exceptional cases also, we shall state and prove the following theorem and show how it applies to the above example.

In what follows, $(H, \Omega, m)$ will denote a regular gage space and $\left(A_{n}\right)$ and $\left(B_{n}\right)$ will stand for a pair of sequences of measurable operators.

(*) $\left(A_{n}\right)$ converges grossly to a measurable operator $A\left(A_{n} \rightarrow A\right.$, grossly, say).

(**) Each $B_{n}$ is non-negative ( $B_{n} \geqq 0$, say), $B_{n}$ in $L^{1}(\Omega, m), B_{n} \rightarrow B$ grossly, $B$ in $L^{1}(\Omega, m)$, and $m\left(B_{n}\right) \rightarrow m(B)$.

We shall discuss some modes of numerical convergence of $\left(A_{n}\right)$, where each $A_{n}$ is dominated by $B_{n}$ in various types.

Theorem 1. If the pair $\left(A_{n}\right),\left(B_{n}\right)$ given by $(*)$ and (**) respectively, satisfy, $\left|A_{n}-A\right| \leqq B_{n}, n=1,2, \cdots$, then $A_{n}-A \rightarrow 0$ in $L^{1}(\Omega, m)$.

Remark. If we assume $B_{n}$ is the same for all $n$, we obtain the dominated convergence theorem of Stinespring [2, Theorem 4.6] as a special case.

Proof. We apply the following version of Fatou's lemma due to Stinespring [2, Theorem 4.10]:-

If $\left(T_{n}\right)$ is a sequence of non-negative measurable operators converging grossly to a measurable operator $T$, then

$$
m(T) \leqq \lim _{n \rightarrow \infty} \inf m\left(T_{n}\right) .
$$

As $A_{n} \rightarrow A$ grossly implies $\left|A_{n}-A\right| \rightarrow 0$ grossly, $S_{n}=B_{n}-\left|A_{n}-A\right| \rightarrow$ $B$ grossly and $S_{n} \geqq 0$.

Now

$$
\begin{aligned}
m(B) & \leqq \liminf _{n \rightarrow \infty} m\left(B_{n}-\left|A_{n}-A\right|\right) \\
& \leqq \lim _{n \rightarrow \infty} m\left(B_{n}-\left|A_{n}-A\right|\right) \\
& \leqq \limsup _{n \rightarrow \infty} m\left(B_{n}\right) \\
& =m(B) .
\end{aligned}
$$

Hence $m\left(B_{n}-\left|A_{n}-A\right|\right) \rightarrow m(B)$; or $m\left(\left|A_{n}-A\right|\right) \rightarrow 0$; or $A_{n}-A \rightarrow 0$ in $L^{1}(\Omega, m)$. This proves Theorem 1 .

The assumption of Theorem 1 is satisfied for the sequences of operators constructed in the previous example. That is, let $A_{n}, P_{n}, \ldots$ etc, be as given in that example. Put $B_{n}=A_{n}+(1-1 / n) /\left(1-1 / 2^{n}\right)$. 
$\left(I-P_{n}\right)$. Clearly, $0 \leqq A_{n} \leqq B_{n}$ for each $n,\left(B_{n}\right)$ converges in measure and hence grossly to $I$ and further $m\left(B_{n}\right)=1 / n+(1-1 / n)=1$.

Corollary 1.1. If $\left(A_{n}\right)$ and $\left(B_{n}\right)$ given by $(*)$ and $(* *)$ respectively satisfy the condition $\left|A_{n}-A\right|^{2} \leqq B_{n}, n=1,2, \cdots$, then $A_{n}-A$ $\rightarrow 0$ in $L^{2}(\Omega, m)$.

To prove this one has only to consider the sequence $\left(S_{n}\right)$ where $S_{n}=B_{n}-\left|A_{n}-A\right|^{2},\left(\left|A_{n}-A\right|^{2}=\left(A_{n}-A\right)^{*}\left(A_{n}-A\right)\right)$, and proceed as in the case of Theorem 1 .

Theorem 2. Let each $A_{n}$ given by $(*)$ be self-adjoint. Suppose that there exist operators $B$ and $B_{n}$ satisfying $(* *)$ such that $\left|A_{n}\right| \leqq B_{n}$, $n=1,2, \cdots$. Then the limit-operator $A$ of $A_{n}$ is self-adjoint, $A$ belongs to $L^{1}(\Omega, m)$ and $m\left(A_{n}\right) \rightarrow m(A)$.

Proof. It has been proved by Stinespring $[1, \mathrm{p} .29]$, that if a sequence $\left(C_{n}\right)$ of non-negative measurable operators converges grossly to a measurable operator $C$, then $C$ is non-nagative. Since for each $n,\left(B_{n} \pm A_{n}\right)$ is non-negative, it follows by the result of Stinespring, that $B$ is non-nagative, $A$ is self-adjoint, and $-B \leqq A \leqq B$. As $B$ is integrable, so is $A$, so that $m(A)$ is finite. If $m\left(A_{n}\right)=m(A)$ for all but finitely many $n$, then there is nothing to prove. Hence, in the most general case, $\left(A_{n}\right)$ consists of two subsequences $\left(A_{i n}\right)$ and $\left(A_{j n}\right)$ such that for all $n, m\left(A_{i n}\right) \geqq m(A)>m\left(A_{j n}\right)$. Let $S_{n}=B_{i n}+|A|-\left(A_{i n}-A\right)$. Each $S_{n}$ is non-negative. And on applying Fatou's lemma,

$$
\begin{aligned}
m(B+|A|) & \leqq \lim _{n \rightarrow \infty} \inf m\left(B_{i n}+|A|-\left(A_{i n}-A\right)\right) \\
& \leqq \lim _{n \rightarrow \infty} \sup m\left(B_{i n}+|A|-\left(A_{i n}-A\right)\right) \\
& \leqq \lim _{n \rightarrow \infty} \sup m\left(B_{i n}+|A|\right) \\
& =m(B+|A|) .
\end{aligned}
$$

Thus as $n \rightarrow \infty, m\left(S_{n}\right) \rightarrow m(B+|A|)$ and $m\left(A_{i n}-A\right) \rightarrow 0$.

For the other part, one need only consider $S_{n}=B_{j n}+|A|-\left(A-A_{j n}\right)$ and proceed as before. Hence the Theorem.

For any measurable operator $T$, put $\operatorname{Re} T=1 / 2\left(T+T^{*}\right)$ and $\operatorname{Im}$ $T=1 / 2 i\left(T-T^{*}\right)$. Applying the proof of Theorem 2 separately to $\left(\operatorname{Re} A_{n}\right)$ and $\left(\operatorname{Im} A_{n}\right)$ in the place of $\left(A_{n}\right)$ given by $(*)$ we have immediately the following

Corollary 2.1. Each $A_{n}$ is dominated by $B_{n}$, satisfying (**) such that $\left|\operatorname{Re} A_{n}\right| \leqq B_{n}$, and $\left|\operatorname{Im} A_{n}\right| \leqq B_{n}, n=1,2, \cdots$. Then $m\left(A_{n}\right) \rightarrow$ $m(A)$.

Now let $\left(A_{n}\right)$ and $A$ satisfy the assumptions of Corollary 2.1. It is worthwhile examining whether $m\left(\left|A_{n}-A\right|\right) \rightarrow 0$ as $n \rightarrow \infty$. The method of proof of Theorem 2 fails to apply even when each $A_{n}$ is self-adjoint. We encounter the following difficulty:- $\left|A_{n}\right| \leqq B_{n}$ for each $n$. Still it does not follow that $\left|A_{n}-A\right| \leqq B_{n}+|A|$, since the 
inequality $\left|A_{n}-A\right| \leqq\left|A_{n}\right|+|A|$ is not in general valid for operators. Hence we cannot conclude that the operator $S_{n}=B_{n}+|A|-\left|A_{n}-A\right|$ is non-negative, so that Fatou's lemma is not applicable to the sequence $\left(S_{n}\right)$.

Acknowledgement. The author is grateful to Prof. Umegaki and Dr. J. Sethuraman for their suggestions towards improving the presentation of this paper.

\section{References}

[1] I. E. Segal: A non-commutative extension of abstract integration. Ann. Math., 57, 401-457 (1953).

[2] W. F. Stinespring: Integration theorems for gages and duality for unimodular groups. Trans. Amer. Math. Soc., 9o, 15-56 (1959). 